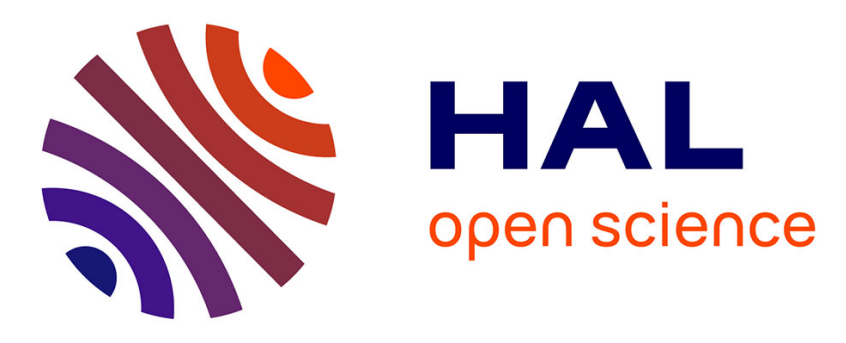

\title{
Reversible magneto-mechanical modeling of heterogeneous media
}

Xingjun Wang, Olivier Hubert, Song He, Frederick-Sorel Mballa-Mballa, Ying Huang

\section{- To cite this version:}

Xingjun Wang, Olivier Hubert, Song He, Frederick-Sorel Mballa-Mballa, Ying Huang. Reversible magneto-mechanical modeling of heterogeneous media. Journal of Superconductivity and Novel Magnetism, 2014, 10.1007/s10948-014-2536-x . hal-01531795

\section{HAL Id: hal-01531795 \\ https://hal.science/hal-01531795}

Submitted on 1 Jun 2017

HAL is a multi-disciplinary open access archive for the deposit and dissemination of scientific research documents, whether they are published or not. The documents may come from teaching and research institutions in France or abroad, or from public or private research centers.
L'archive ouverte pluridisciplinaire HAL, est destinée au dépôt et à la diffusion de documents scientifiques de niveau recherche, publiés ou non, émanant des établissements d'enseignement et de recherche français ou étrangers, des laboratoires publics ou privés. 


\title{
Reversible magneto-mechanical modeling of heterogeneous media
}

\author{
Xingjun $\mathrm{WANG}^{1,2^{*}}$, Olivier HUBERT ${ }^{3}$, Song $\mathrm{HE}^{2}$, Frederick-Sorel MBALLA-MBALLA ${ }^{3}$, Ying \\ $\mathrm{HUANG}^{4}$
}

1 Department of Physics and Electronic Science, Qiannan Normal College for Nationalities, Guizhou 558000, China

2 Pôle Universitaire Léonard de Vinci, 12 Avenue Léonard de Vinci, 92400 Courbevoie, France

3 LMT-Cachan (ENS-Cachan/UMR CNRS 8535/UPMC/Pres Universud Paris) 61, avenue du président Wilson 94235 Cachan cedex, France

4 Department of Mechanical Engineering, Taizhou University, Taizhou 318000, China

\begin{abstract}
Localization and homogenization of the magneto-mechanical fields in heterogeneous media are discussed. The magneto-elastic modeling for magnetic polycrystals is extended from the description of the single crystal modeling. This model is then applied to the prediction of the behavior of dual-phase steels. Each phase can be considered as a sphere embedded in a homogeneous equivalent medium. The magnetic model used for each phase is an explicit single crystalline model representative of the behaviour of the corresponding phase. Homogenization rules allow the simulation of the average medium. Experiments are carried out and compared to the numerical results.
\end{abstract}

Keywords: Ferromagnetic materials, DP steels, magneto-mechanical modeling, localization.

\section{Introduction}

Non destructive evaluation (NDE) is used in the industries of steel since magnetic behavior demonstrates a good sensitivity to phase fraction of different nature, applied stress, residual stresses and plasticity. Many researchers have devoted themselves to developing higher performance modeling in order to implement inverse identification, ie predict the thermo-metallurgico-mechanic state of a material from a simple magnetic measurement.

On line NDE is particularly an important point, in order to optimize on line parameters of a forming or elaborating process. Models should take on the other hand account for the multiphased state of real materials and for the coupling with mechanical stress. This coupling is characterized by the influence of stress on the magnetic susceptibility and by magnetostriction. The magnetostriction $\varepsilon^{\mu}$ consists in a dilatation or a contraction depending on the applied field $\vec{H}$ level and direction [1]. This deformation is sensitive to any change of the material microstructure or applied stress $[2,3]$. The effect of mechanical stress $\boldsymbol{\sigma}$ on magnetization $\bar{M}$ of a material (change in magnetic susceptibility) is a second manifestation of the magneto-mechanical coupling. In case of uniaxial stress, the effect of tensile or compressive stress is usually not symmetric [2]; it depends on material composition, magnetic field and stress levels. Multiaxiality of stress can on the other hand play a significant role [4]. At higher stress

\footnotetext{
* To whom correspondence should be addressed. Tel.:+86 15825472448 .

E-mail:xingjun.wang@gmail.com
} 
levels, plastic straining occurs, leading to a sharp degradation of the magnetic properties (strong decrease of susceptibility, increase of hysteresis losses) [5] and a change in the magnetostriction amplitude [6]. Classical magnetoelasticity is able to explain these phenomena assuming that the driving force associated to plasticity is the long range internal stresses $[6,7]$.

The modeling of magneto-mechanical phenomena is usually done using an energetic approach where variables are written at an appropriate scale. On the one hand, several macroscopic models have been proposed. The applicability of these approaches is limited to a short range of loadings, isotropic materials and rarely accounted for multiaxial stress. These models do not take account of the microstructure of the material; magneto-mechanical coupling is consequently partially described. The development of micromagnetic or fully multiscale magneto-elastic models is a promising issue $[8,9]$. Because the number of degrees of freedom and interactions is increasing quickly with the number of magnetic moments, these simulations are always used for small size systems. When a complex heterogeneous material is considered, these models lead to dissuasive computation times. Inverse identification is consequently still not reachable.

As part of Nondestructive testing (NDT), a fast accurate modeling to estimate the magneto-elastic behaviour has become a crucial element to be taken into account. Our objective is to combine the accuracy of micromagnetic modeling in order to describe complex magneto-mechanical phenomena, to the simplicity and speed of macroscopic approaches. Quantitative inverse identification should be reached using few experiments.

The paper is divided into four parts. In the first part, localization and homogenization rules are discussed, which allow to define residual (internal) stress tensor and local demagnetizing field. The second part presents the single-crystalline magnetomechanical model. In the third part, specific numerical implementation for the magnetic field is detailed: a method using Taylor series for heterogeneous field is proposed. In the last part, the proposed model is used to simulate a dual-phase steel- polycrystalline and multiphased medium-, where heterogeneity is associated to the anisotropic behavior of the single crystal and to the nature of the crystal (ie ferrite / martensite). The modeling considers homogeneous magnetic field and strain within the single crystal. Hysteretic phenomena are not considered. The proposed modeling is associated to anhysteretic magnetic field strengthening and can be developed for ferri- or ferromagnetic materials.

\section{Localization and homogenization of the magneto-mechanical fields in}

\section{Heterogeneous media}

The representative volume element (RVE) is seen as an assembly of volume of interest (VOI). VOI is for example a grain inside a polycrystal or an assembly of grains. The problem will be solved (calculation of magnetic, mechanical and coupled quantities) at the VOI scale. The different VOIs have to communicate with each others. Localization (expression of local loadings as function of global loadings) and homogenization (averaging) operations can be made. 


\subsection{Localization and homogenization of the magnetic fields in heterogeneous} media

The aim of this step consists in deriving the local magnetic field $\vec{H}_{I}$ from the external loading ( magnetic field $\mathrm{H}$, and stress $\Sigma$ ), $\vec{H}$ postulating a given form for the function $h$ :

$$
\vec{H}_{I}=h\left(\sum, \vec{H}\right)
$$

This equation is usually written (in electrotechnical engineering) in the form

$$
\vec{H}_{I}=\vec{H}+\vec{H}_{d}
$$

where the local perturbation of the macroscopic magnetic field is taken into account through the demagnetising field $\vec{H}_{d}$. The general form of the localization law can be written:

$$
\vec{H}_{I}-\vec{H}=\boldsymbol{L}\left(\vec{M}-\vec{M}_{I}\right)
$$

with $\bar{M}$ the mean magnetization in the material, $\bar{M}_{I}$ the magnetization of the VOI. $\boldsymbol{L}$ is a 2 nd order localization operator, depending on the magnetization, on the stress state, and on the shape choosen for the inclusion $I$. In the case of stress independent linear isotropic magnetic behavior, and spherical inclusions, the tensor $L$ reduces to scalar value $N$ usually called demagnetizing factor so that:

$$
N=\frac{1}{3+2 \chi}
$$

with $\chi$ the equivalent medium susceptibility. We get

$$
\vec{H}_{I}=\vec{H}+\frac{1}{3+2 \chi}\left(\vec{M}-\vec{M}_{I}\right)
$$

This approach demonstrated for linear medium has been extended with success to anisotropic non-linear magnetic behavior [9] using:

$$
\chi=\frac{M}{H}
$$

Formulation 5 becomes self-consistent. Its numerical implementation remains a challenge. A new algorithm is proposed further in the text, avoiding a step by step calculation.

\subsection{Localization and homogenization of the mechanical fields in heterogeneous media}

The aim of this step consists in deriving the local stress $\boldsymbol{\sigma}$ from the external loading (magnetic field $\mathrm{H}$, and stress $\Sigma$ ), postulating a particular form for the function $g$ in:

$$
\sigma_{I}=g(\Sigma, \vec{H})
$$

The function $g$ is here deduced from a self-consistent approach. Each inclusion is considered imbedded in a homogeneous equivalent medium equivalent so that the problem can be linked to the solution of the Eshelby inclusion problem [10]. 
Consider an unloaded homogeneous infinite medium of stiffness tensor $\mathbb{C}$. A VOI is submitted to a free magnetostriction strain $\varepsilon_{I}^{\mu}$. This strain is the strain that would act in the VOI if no stress was exerted by the surrounding medium. The actual strain $\varepsilon_{I}$ in the VOI can be linked to the free strain using the Eshelby tensor $\mathbb{S}^{E}[10]$ :

$$
\varepsilon_{I}=\mathbb{S}^{E}: \varepsilon_{I}^{\mu}
$$

The fourth order tensor $\mathbb{S}^{E}$ only depends on the stiffness tensor $\mathbb{C}$ and the shape chosen for the VOI. It is calculated from the Green's functions and FDO [9].

The stress in the VOI $\varepsilon_{I}$ is related to the elastic strain of the VOI thanks to the Hooke's law:

$$
\sigma_{I}=\mathbb{C}_{I}: \varepsilon_{I}^{e}
$$

Considering the strain decomposition, this decomposition is acceptable only when deformation levels are low $\left(<10^{-2}\right)$, we get:

$$
\varepsilon_{I}=\varepsilon_{I}^{e}+\varepsilon_{I}^{\mu}
$$

The stress can be expressed as function of the free strain:

$$
\sigma_{I}=\mathbb{C}_{I}:\left(\varepsilon_{I}-\varepsilon_{I}^{\mu}\right)
$$

Considering an applied stress $\sum$ over the VOI, the stress at the grain scale $\sigma^{g}$ is derived from the implicit equation (13).

$$
\begin{gathered}
E_{\mu}=<{ }^{t} \mathrm{~B}: \varepsilon_{\mu}^{g}> \\
\sigma_{I}=\mathrm{B}: \Sigma+\mathbb{C}^{a c c}:\left(E^{\mu}-\varepsilon_{I}^{\mu}\right)
\end{gathered}
$$

With $\mathbb{C}^{a c c}=\left(\mathbb{C}_{I}\right)^{-1}+\left(\mathbb{C}^{0}:\left(\left(S^{E}\right)^{-1}-\mathrm{I}\right)\right)^{-1} \cdot \mathbb{C}^{0}$ is the stiffness tensor of the effective media[11]. Since a self-consistent scheme has been chosen, $\mathbb{C}^{0}$ refers to the self-consistent stiffness tensor [9]. B is the so-called stress concentration tensor.

\section{Multidomain magneto-mechanical modeling of single-crystals}

The prediction of the influence of heterogeneity on magnetic behavior supposes the introduction of the complete mechanical loading into a magnetoelastic modeling. As already mentioned, the few practically implemented models describing the effect of stress on magnetic behavior are restricted to uniaxial mechanical loadings (tension or compression) [12]. A first approach to build a simple magneto-elastic model consists in the definition of magneto-elastic constitutive laws including the multiaxiality of stress at the local scale. A multidomain modeling following this requirement is briefly presented.

\subsection{Original formulation}

The multidomain modeling is a two-scale reversible modeling allowing the prediction of the 
magneto-mechanical behavior of isotropic polycrystals. It comes from a simplification (which is simplified in four points: simplified microstructure, homogeneous field and stress, uniaxial, stress, no minimization of energy.) of the so-called multiscale model [9]. A six magnetic domains configuration is considered associated to the six easy axes of cubic symmetry for materials that exhibit a positive magnetocrystalline constant (figure 1a). Each domain family $\alpha$ is defined by a magnetization vector $\vec{M}_{a}$ so that $\left\|\vec{M}_{a}\right\|=M_{s}$, and by a magnetostriction tensor $\varepsilon_{a}^{\mu}$ (15) $\left(\gamma_{\mathrm{i}}\right.$ parameters figure the direction cosines of magnetization; $\lambda_{100}$ and $\lambda_{111}$ are the two magnetostrictive constants). This single crystal is considered as submitted to a magnetic field $\vec{H}$ and/or stress $\sigma$. Uniform strain and field hypotheses are used over the crystal and domain walls contribution to the total energy is neglected [9]. The energy of a magnetic domain $W_{a}$ is the sum of the magnetostatic energy $W_{a}^{H}$, the magneto crystalline energy $W_{a}^{K}$ and of the magnetoelastic energy $W_{a}^{\sigma}(16)\left(\mathrm{K}_{1}\right.$ is the magneto crystalline constant of the material). The stress tensor is supposed uniaxial; magnetic field and stress are applied along a same direction $\vec{n}_{c}$ defined by angles $\phi_{c}$ and $\theta_{c}$ of the spherical frame (figure $1 \mathrm{~b}$ ). This direction is restricted to the standard triangle defined by crystallographical directions $<100>,<110>$ and $<111>$ : cubic symmetry means that at any loading direction is corresponding a direction in this triangle. The resolution of the problem (ie calculation of the mean magnetization and deformation) requires to evaluate the direction of magnetization and the volumetric fraction of each domain family $\alpha$.

$$
\varepsilon^{\mu}=\frac{3}{2}\left(\begin{array}{lll}
\lambda_{100}\left(\gamma_{1}^{2}-\frac{1}{3}\right) & \lambda_{111} \gamma_{1} \gamma_{2} & \lambda_{111} \gamma_{1} \gamma_{3}\left[\gamma_{1}, \gamma_{2}, \gamma_{3}\right] \\
\lambda_{111} \gamma_{1} \gamma_{2} & \lambda_{100}\left(\gamma_{2}^{2}-\frac{1}{3}\right) & \lambda_{111} \gamma_{2} \gamma_{3} \\
\lambda_{111} \gamma_{1} \gamma_{3} & \lambda_{111} \gamma_{2} \gamma_{3} & \lambda_{100}\left(\gamma_{3}^{2}-\frac{1}{3}\right)
\end{array}\right)
$$

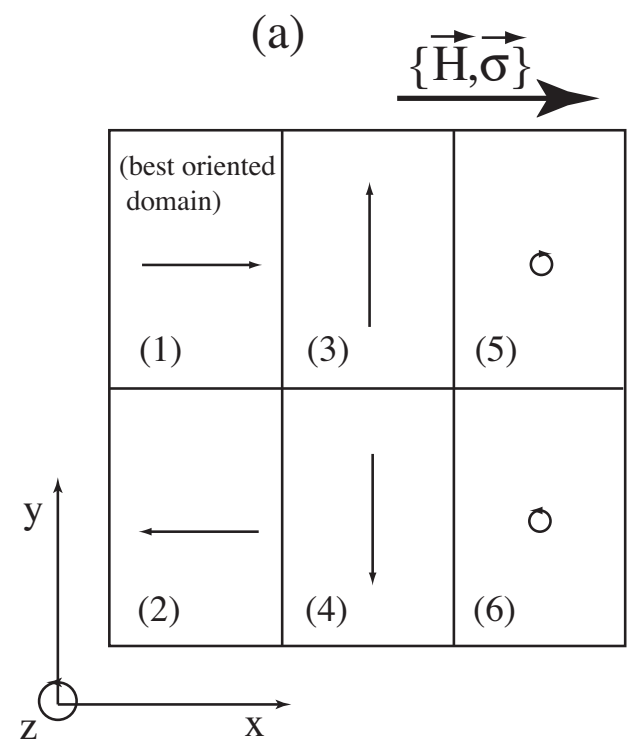

(a) (b)

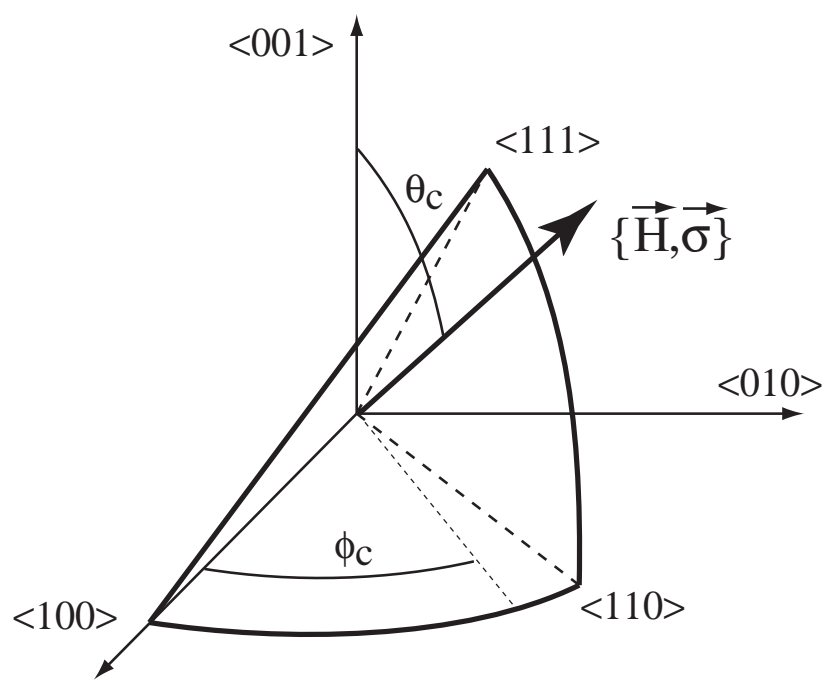

(b) 
Fig. 1. (a) Initial domain structure of single crystal considered for the multidomain modeling; (b) Standard triangle and parameters for magneto-mechanical loading axis.

$$
\begin{aligned}
& W_{a}^{H}=-\mu_{0} \vec{H} \cdot \vec{M}_{a} \\
& W_{a}^{\sigma}=-\sigma: \varepsilon_{a}^{\mu} \\
& W_{a}^{K}=K_{1}\left(\left(\gamma_{1} \gamma_{2}\right)^{2}+\left(\gamma_{2} \gamma_{3}\right)^{2}+\left(\gamma_{1} \gamma_{3}\right)^{2}\right)
\end{aligned}
$$

The volumetric fraction $f_{a}$ of a domain is calculated thanks to statistical Boltzmann formula (18);

$A_{s}$ is an adjusting parameter corresponding to the inverse of entropy following Boltzmann formalism.

A simple Taylor expansion of volumetric fraction at low field is showing that $A_{s}$ is proportional to the initial susceptibility $\chi_{0}$ of the magnetization curve. We get: $A_{s}=\frac{3 \chi_{0}}{\mu_{0} \cdot M_{s}^{2} \cdot\left(\cos \phi_{c} \sin \theta_{c}\right)^{2}}$.

$$
\begin{array}{|c|c|}
\vec{n}_{c}={ }^{t}\left[\cos \left(\phi_{c}\right) \sin \left(\theta_{c}\right), \sin \left(\phi_{c}\right) \sin \left(\theta_{c}\right), \cos \left(\theta_{c}\right)\right] & \text { (17) } \\
\hline f_{a}=\frac{\exp \left(-A_{s} \cdot W_{a}\right)}{\sum_{a} \exp \left(-A_{s} \cdot W_{a}\right)} & \\
\hline\left[\gamma_{1}, \gamma_{2}, \gamma_{3}\right]=\left[\cos \left(\phi_{a}\right) \sin \left(\theta_{a}\right), \sin \left(\phi_{a}\right) \sin \left(\theta_{a}\right), \cos \left(\theta_{a}\right)\right] & \text { (19) } \\
\hline
\end{array}
$$

The magnetization direction of a domain is defined by angles $\phi_{a}$ and $\theta_{a}$ of the spherical frame (19). These angles are usually estimated thanks to a minimization of the potential energy (20). In the present study, the restriction to standard triangle allows an analytical minimization, so that a constitutive law for the angles of each domain is obtained as function of magnetic field, stress, and loading direction parameters. As an example, equations (21) give the constitutive laws of the two spherical angles for domain $\alpha=1$ (Cf. figure 1a).

$$
\begin{aligned}
& \frac{d W_{a}}{d \phi_{a}}\left(H, \sigma, \phi_{c}, \theta_{c}, \phi_{a}, \theta_{a}\right)=0 \\
& \frac{d W_{a}}{d \theta_{a}}\left(H, \sigma, \phi_{c}, \theta_{c}, \phi_{a}, \theta_{a}\right)=0
\end{aligned}
$$




$$
\begin{aligned}
\phi_{1}\left(H, \sigma, \phi_{c}\right) & =\frac{\mu_{0} M_{s} H \phi_{c}+a \tan \left(\frac{3}{2} \lambda_{111} \sigma \sin \left(2 \phi_{c}\right)\right)}{\mu_{0} M_{s} H+2 K_{1}+3 \lambda_{100} \sigma \cos \left(2 \phi_{c}\right)} \\
\theta_{1}\left(H, \sigma, \theta_{c}\right) & =\frac{\mu_{0} M_{s} H\left(\frac{\pi}{2}-\theta_{c}\right)+a \tan \left(\frac{3}{2} \lambda_{111} \sigma \sin \left(2\left(\frac{\pi}{2}-\theta_{c}\right)\right)\right)}{\mu_{0} M_{s} H+2 K_{1}+3 \lambda_{100} \sigma \cos \left(2\left(\frac{\pi}{2}-\theta_{c}\right)\right)}
\end{aligned}
$$

Average magnetization and magnetostriction (22) are projected along the loading axis $\vec{n}_{c}$ leading to the behavior of the single crystal $M(H, \sigma)$ and $\varepsilon_{/ /}^{\mu}(H, \sigma)(23)$.

$$
\begin{array}{cc}
\vec{M}=\sum_{a} f_{a} \vec{M}_{a} & \mathcal{E}^{\mu}=\sum_{a} f_{a} \varepsilon_{a}^{\mu} \\
M & =\vec{M} \cdot \vec{n}_{c} \quad \varepsilon_{/ /}^{\mu}=\stackrel{t}{n}_{c} \cdot \mathcal{E}^{\mu} \cdot \vec{n}_{c}
\end{array}
$$

\subsection{Extension to loading out of the standard triangle}

Multi-domain single-crystal model can be employed only when the loading is applied in the standard triangle. Thus, the local loading (Magnetic field and stress) need a linear transformation in order to mach the standard triangle. A permutation matrix is used to make the load to be adapted for a symmetries cubic crystal.

Since the standard triangle is a so-called spherical triangle defined between $\langle 100\rangle,\langle 110\rangle$ and $\langle 111\rangle$ crystallographic directions, 48 triangles are required to map the whole surface of the sphere.

Let consider $\vec{n}_{c}^{i}$ the loading direction inside triangle $T_{i} \cdot \vec{n}_{c}$ is the loading direction inside the standard triangle $T_{1}$ corresponding to $\vec{n}_{c}^{i}$, meaning leading to the same behavior that a loading along $\vec{n}_{c}^{i}$ due to cubic symmetry of the single crystal. $P_{i}$ is the corresponding permutation matrix verifying:

$$
\vec{n}_{c}=P_{i} \cdot \vec{n}_{c}^{i}
$$

48 permutation matrix can consequently be defined, allowing to define a loading direction inside $T_{1}$

triangle whatever the loading direction. $P_{1}$ is the permutation matrix from triangle $T_{1}$ to triangle $T_{1}$ $\left(\vec{n}_{c}=\vec{n}_{c}\right) ; P_{2}$ is the permutation matrix from triangle $T_{2}$ to triangle $T_{1} ;$ etc..., leading to: 


$$
\begin{aligned}
& p_{1}=\left(\begin{array}{lll}
1 & 0 & 0 \\
0 & 1 & 0 \\
0 & 0 & 1
\end{array}\right)=P_{1 \rightarrow 1}=I \\
& p_{2}=\left(\begin{array}{lll}
0 & 1 & 0 \\
1 & 0 & 0 \\
0 & 0 & 1
\end{array}\right)=P_{2 \rightarrow 1}
\end{aligned}
$$

The permutation must be applied to magnetic field and uniaxial stress direction and so on, so that:

$$
\begin{aligned}
& \vec{H}=P^{i} \cdot \vec{H}^{i} \\
& \vec{\sigma}=P^{i} \cdot \vec{\sigma}^{i}
\end{aligned}
$$

The constitutive law is applied then Magnetic and magnetostrictive quantities are calculated using multidomain modeling. Results must be submitted to a reversal permutation in order to get the magnetization and magnetostriction tensor in the appropriate frame.

$$
\begin{aligned}
\vec{M}^{i} & =P^{i-1} \cdot \vec{M} \\
\varepsilon^{\mu i} & =P^{i-1} \cdot \varepsilon^{\mu} P^{i}
\end{aligned}
$$

\subsection{Extension to multiaxial stress}

The second step is to calculate stress used for rotation. We change the multiaxial stresses $\boldsymbol{\sigma}$ into uniaxial magneto-mechanical equivalent stresses according to the direction of the magnetic loading $\vec{n}_{c}$. We use for that purpose an equivalent stress recently defined in [13]. In the frame $\left(\vec{h}, \vec{t}_{1}, \vec{t}_{2}\right)$ associated to the magnetic field direction $\vec{h}$ and two orthogonal directions associated to the matter symmetries. We note $k$ the product $A_{s} \lambda_{m}$ as a material dependent parameter:

$$
\sigma_{e q}=\vec{h} S \vec{h}-\frac{2}{3 k} \ln \left[\frac{\exp \left(\frac{3 k}{2} \vec{t} \vec{t}_{1} S \overrightarrow{t_{1}}\right)+\exp \left(\frac{3 k}{2} \vec{t}_{2} S \overrightarrow{t_{2}}\right)}{2}\right]
$$

$\mathrm{S}$ is the deviatoric tensor associated to $\sigma$. Using the hypothesis of uniform stress, we get

$$
k=\frac{6 \chi_{0} \lambda_{100}}{5 \mu_{0} M_{s}^{2}}
$$

for positive magneto crystalline anisotropy materials and

$$
k=\frac{9 \chi_{0} \lambda_{111}}{5 \mu_{0} M_{s}^{2}}
$$

for negative magneto crystalline anisotropy materials. $\chi_{0}$ denotes the initial anhysteretic susceptibility 
of the material, $\mu_{0}$ is the permeability of vacuum and $M_{s}$ is the saturation magnetization of the material. This definition of equivalent stress finally requires to know some relatively usual material parameters and no supplementary adjusting parameter. This expression can be degraded in a more simple expression (deviatoric equivalent stress (31)) applicable to moderate loadings and/or relatively low magnetostrictive materials.

$$
\sigma^{e q}=\frac{3}{2} \stackrel{t \rightarrow i}{n_{c}} \overrightarrow{S n}_{c}^{i}
$$

The same value of equivalent stress is considered to be applied along $\vec{n}_{c}$. We remark that permutation operation can be made on the stress tensor, so we have:

$$
\begin{gathered}
\vec{n}_{c}^{i}=P^{i-1} \cdot \vec{n}_{c} \\
\sigma^{e q}=\frac{3}{2}{ }^{t} \vec{n}_{c}^{t} P^{i-1} S P^{i-1} \cdot \vec{n}_{c} \\
\sigma^{e q}=\frac{3}{2} \vec{n}_{c} S^{\prime} \cdot \vec{n}_{c}
\end{gathered}
$$

The same operation can be applied to the generalized formulation of equivalent stress.

\subsection{Single crystal modeling}

For a single iron crystal, it is possible to subdivide the volume in six domain families associated with the six easy magnetization directions. Each domain family is defined by a specific magnetization and magnetostriction strain tensor. Considered loadings are 3D multiaxial stress and/or magnetic field. Orientation of magnetization of each domain defined analytically avoiding energetic minimization: use of equivalent axial loading inside the standard triangle. Volume fraction of domains is calculated thanks to energy consideration [14].

\section{Multidomain magneto-mechanical modeling of polycrystals}

This method is applied to calculate the average behavior of polycrystalline medium.

\subsection{Orientation Distribution Function - ODF}

We consider in a first approach an isotropic texture represented by an orientation distribution function at 546 discrete grains oriented to pave properly the entire sphere surface. Euler angles are as:

TABLE 1 Euler angles of isotropic polycrystal of 546 grains

\begin{tabular}{|c|c|c|}
\hline Euler angles & Domain & $\begin{array}{c}\text { Number of } \\
\text { values }\end{array}$ \\
\hline$\phi_{1}$ & {$[0 ; 2 \pi]$} & 13 \\
\hline $\cos \psi$ & {$[0 ; \pi]$} & 7 \\
\hline
\end{tabular}




\begin{tabular}{|l|l|l|}
\hline$\phi_{2}$ & {$[0 ; 2 \pi]$} & 6 \\
\hline
\end{tabular}

The following figure (Fig. 2) shows the associated pole figures. Each grain orientation is defined by a matrix $\mathrm{Q}$ providing the transition of local frame to macroscopic frame $(\mathrm{x}, \mathrm{y}, \mathrm{z})$. We can thus define a local orientation of the load.
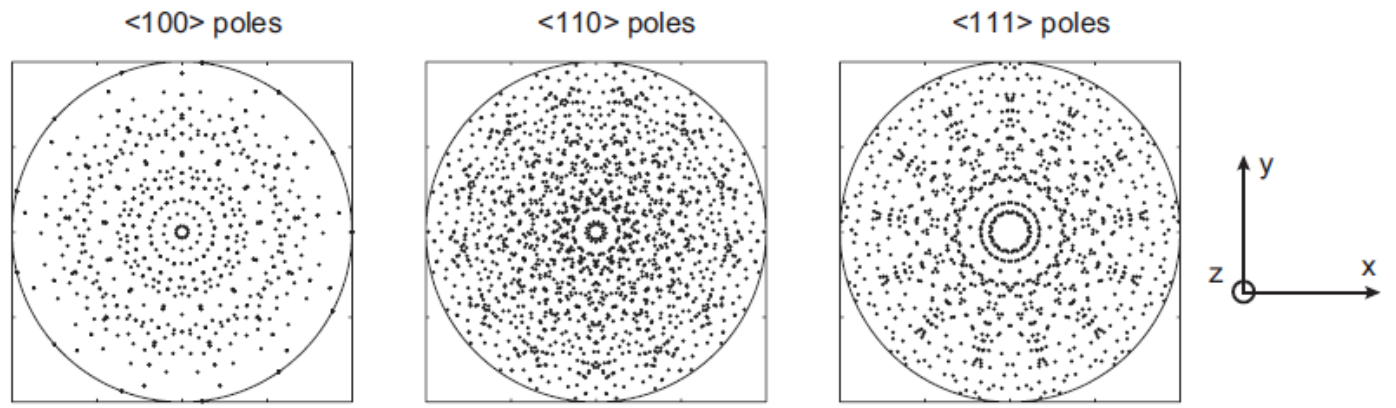

Fig. 2. Pole figure associated with the distribution function of orientations 546 discrete grains uniformly distributed in space

We perform the following operations:

1) Transition of vector fields from the macroscopic frame to the local frame: localization.

2) Transition of vector fields from the local frame (sphere) to the standard triangle by appropriate permutation.

3) Application of constitutive law; Calculation of the local magnetization and magnetostriction.

4) Passage of the magnetization vector of the local landmark macroscopically.

5) Calculation of mean fields and magnetizations of the 546 directions.

The first method uses the implicit nature of the formulation of field location.

Assuming the magnetic field is known to each grain $H_{I}$, one can use the following operations to determine the magnetization field in each grain $M_{I}$ and the mean fields $M$.

The problem is proposed as following: for a given external magnetic field $H$, we can define the magnetization field $M_{I}$ and the magnetic field $H_{I}$ for each grain, there is two relations between $M_{I}$ and $H_{I}$ : on one part, once $M_{I}$ is known, $H_{I}$ can be determined using Eq. (5), on the other hand, $H_{I}$ can be known if the process above is used, then $M_{I}$ can be calculated. For simplify the notation, the relation is denoted:

$$
M_{I}=F\left(H_{I}\right)
$$

Where $\mathrm{F}$ is a function that depends the transition matrices $\mathrm{Q}$ and can be determined explicitly.

But in practice, it is difficult to give the analytical formula for F. By consequence, $M_{I}$ and $H_{I}$ can not be determined analytically. Thus, an iterative process is proposed to determine their values. Assuming that $M^{(n)}$ is known at the Nth iteration, noted $M_{I}^{(n)}$, we can determine 
- The magnetic field $H_{I}^{(n)}$ using the relation (35);

- The magnetization field $M_{I}^{(n)}$ using the relation (5).

So, iteration will continue until the difference between these two iterations is considered sufficiently small. Combining these two equations above (Eq. (35) and Eq. (5)), we can get

$$
M_{I}^{(n+1)}=F\left(H+\frac{1}{3+2 \chi}\left(M^{(n)}-M_{I}^{(n)}\right)\right),
$$

Where $M^{(n)}$ (the mean magnetic field $M_{I}^{(n)}$ ) also depends on $M_{I}^{(n)}$. Thus, the formula can be simplified as

$$
M_{I}^{(n+1)}=G\left(M_{I}^{(n)}\right)
$$

Where the function can be determined by F, H and $\chi$; and we go back to a problem of fixed point. As we mentioned above, the convergence property of the algorithm depends on the quality of function $\mathrm{G}$. In fact, $\mathrm{G}$ is needed to be contractive, i.e.

$$
\|G(x)-G(Y)\| \leq L\|X-Y\| \quad(\text { where } L<1)
$$

Satisfying at least for $\mathrm{X}$ and $\mathrm{Y}$ in the vicinity of the solution $M_{I}$.

$\mathrm{G}$ is a non-analytical function; it is difficult to verify this contracting condition theoretically. For this reason, we use the numerical verification. One state of $M_{I}$ is chosen and a little bit of disturbing is done for $\left(M_{I}^{*}\right)$, then the numerical value of $\left(\left\|G\left(M_{I}\right)-G\left(M_{I}^{*}\right)\right\|\right) /\left(\left\|M_{I}-M_{I}^{*}\right\|\right)$ is calculated. For reason of computational complexity, three grains are considered to simply the case. As we do not know the state of the equilibrium of the magnetization field $M_{I}$, many cases $(>8000)$ have been tested in order to cover all of the possible configurations of $M_{I}$.

We notice that the values of $\left(\left\|G\left(M_{I}\right)-G\left(M_{I}^{*}\right)\right\|\right) /\left(\left\|M_{I}-M_{I}^{*}\right\|\right)$ are superior to 1 in many cases, so that the convergence of algorithm is not ensured. Some implemented tests have confirmed our fears: the algorithm does not provide convergence except for the case where only two grains are considered. The usual proposition to avoid this problem is to use an incremental procedure. We consider two steps of magnetic field denoted $H^{(n)}$ and $H^{(n+1)}$. The local magnetic behavior at step $(\mathrm{n}+1)$ is given by macrocropic field at the same step and demagnetizing field calculated at the previous step (n) following equation (5). We get:

$$
\vec{H}_{I}^{(n+1)}=\vec{H}^{(n+1)}+\frac{1}{3+2 x^{(n)}}\left(\vec{M}^{(n)}-\vec{M}_{I}^{(n)}\right)
$$

Average condition is still verified 


$$
\vec{H}^{(n+1)}=<\vec{H}_{I}^{(n+1)}>
$$

We propose in the next subsection a new formulation allowing a true calculation of the demagnetizing field.

\subsection{Algorithms}

The Taylor series is a representation of a function as an infinite sum of terms calculated from the values of its derivatives at a single point. The Taylor series can be defined in the following manner.

$$
\begin{aligned}
& f(x)=f(a)+\frac{f^{\prime}(a)}{1 !}(x-a)+\frac{f^{\prime \prime}(a)}{2 !}(x-a)^{2} \\
& +\frac{f^{(3)}(a)}{3 !}(x-a)^{3}+\ldots=\sum_{n=0}^{\infty} \frac{f^{(n)}(a)}{n !}(x-a)^{n}
\end{aligned}
$$

where $n$ ! denotes the factorial of $n$ and $(n)$ denotes the $n^{\text {th }}$ derivative of $\mathrm{f}$ evaluated at the point a. Assuming the first and second right terms of equation (39) are much bigger than the other terms, equation (40) is obtained

$$
f(x)=f(a)+\frac{f^{\prime}(a)}{1 !}(x-a)
$$

On the other hand, the localization law is considered (corresponding to the 3 projections $(\mathrm{k})$ of equation):

$$
\left(H_{j}^{i+1}=H^{i}+\frac{1}{3+2 \chi^{i}}\left(M^{i}-M_{j}^{i}\right)\right) k
$$

where $j$ is the index of grains, $i$ is the number of loop for localization (not corresponding to magnetic field step). $M^{i}$ is the average magnetization for all the grains at iteration $i$ so that

$$
M^{i}=<M_{j}^{i}>
$$

and $\chi^{i}$ is the mean secant susceptibility given by

$$
\chi^{i}=\left\|\vec{M}^{i}\right\| /\left\|\vec{H}^{i}\right\|
$$

Convergence is achieved when

$$
\left(H_{j}^{i+1}-H_{j}^{i}\right)_{k} \rightarrow 0
$$

The magnetization $M_{j}$ is on the other hand function of the magnetic field $H_{j}$ thanks to local constitutive behavior $F$ so that:

$$
\left(M_{j}^{i}=F\left(H_{j}^{i}\right)\right) k \quad \text { or } \quad\left(M_{j}^{i+1}=F\left(H_{j}^{i+1}\right)\right) k
$$

Using equation (41), the constitutive law gives: 


$$
\left(M_{j}^{i+1}=F\left(H^{i}+\frac{1}{3+2 x^{i}}\left(M^{i}-M_{j}^{i}\right)\right)\right) k
$$

When the loop step $i$ turns to infinite, the magnetization should be a stable value. The magnetization of $i+1^{\text {th }}$ loop step equals to $i^{\text {th }}$ loop step magnetization.

$$
M^{i+1}=M^{i}
$$

From equation (44) and (43) the magnetization for $\mathrm{j}$ grain is obtained:

$$
\left(M_{j}=F\left(H+\frac{1}{3+2 x}\left(M-M_{j}\right)\right)\right) k
$$

Because $M$ and $\chi$ are function of $M_{j}, H$ being a constant for a given loop, we get the following self-consistent relation:

$$
\left(M_{j}=G\left(M_{j}\right)\right) k
$$

We consider now a Taylor expansion of $G$ function:

$$
\left(M_{j}^{(n+1)}=G\left(M_{j}^{(n)}\right)+G^{\prime}\left(M_{j}^{(n)}\right)\left(M_{j}^{(n+1)}-M_{j}^{(n)}\right)\right) k
$$

where $(n)$ indicates the $n_{t h}$ magnetic field step. It is assumed that $G$ function is sufficiently linear between two magnetic field step and so two magnetization steps. Equation (47) is rewritten:

$$
\left(M_{j}^{(n+1)}=\frac{G\left(M_{j}^{(n)}\right)-G^{\prime}\left(M_{j}^{(n)}\right) M_{j}^{(n)}}{1-G^{\prime}\left(M_{j}^{(n)}\right)}\right) k
$$

The matrix inversion increases considerably the computation time if 546 orientations are considered. Please note that a calculation field takes about 20 minutes with a laptop and a trade algorithm coded in MATLAB. The taylor expansion is applied to the "local" behavior in the study.

\section{Application to dual-phase steel}

Dual-Phase steels are produced thanks to a complex forming process that leads to a microstructure exhibiting two phases (fig. 3). The morphology and the distribution of the martensite islands in the ferritic matrix are the keys of the dual phases steel behaviour. This microstructure is obtained by quenching of a low carbon steel ( $\mathrm{wt} \% \mathrm{C}<0.3 \%$ typically) from austeno-ferritic domain of the phase diagram.

\subsection{Dual-phase ferrite-martensite steel}

The magneto-mechanical behaviour of a dual phase steel is deeply linked to its microstructure and especially to the behaviour of its constituents. An accurate modeling of the mean medium supposes consequently to have an accurate modeling of each phase separately. The behaviour of ferrite is supposed very close to the behaviour of pure iron, which was the subject of many works. The measurements presented hereafter have been carried out using an Armco pure iron sample. 
Table 2. Chemical composition of DP steel used for the study.

\begin{tabular}{|l|l|l|l|l|l}
\cline { 2 - 6 } \multicolumn{1}{c|}{} & $\mathrm{C}$ & $\mathrm{Mn}$ & $\mathrm{S}$ & $\mathrm{N}$ & $\ldots$ \\
\hline $\mathrm{ppm}$ & 1461 & 18852 & 14 & 178 & $\ldots$
\end{tabular}

The behaviour of martensitic phase is still unknown. Tab. 2 gives the chemical composition of the DP steel used for the study. The carbon content is close to $0.15 \mathrm{wt} \%$. Figure 3 gives the typical microstructure observed for the material as quenched. The volume fraction of martensite has been evaluated to $40 \%$. Assuming that carbon content of ferrite is about $0.02 \mathrm{wt} \%$, the carbon content in the martensite phase can be so evaluated to $0.4 \mathrm{wt} \%$.
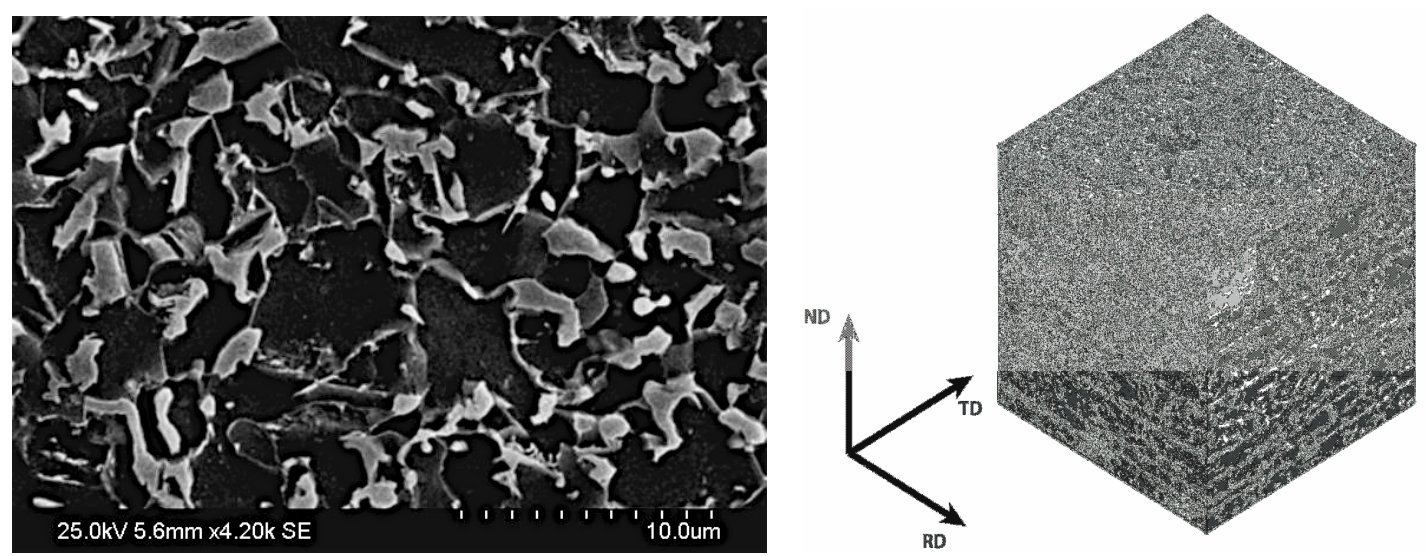

Fig. 3. Dual phase steel microstructure: distribution of martensite in white, and ferrite in dark. 1) Behavior and modeling of the single crystal of martensite: A C38 carbon steel has been considered for this step ( $w \mathrm{t} \% \mathrm{C}=0.38 \%$ ). It has been submitted to various heat treatment (quenching) in order to transform the initial microstructure (fig. 4a) into a microstructure close to the microstructure of the second phase of the dual phase steel. Hardness measurements are used as indicator of the quality of the new microstructure. Figure $4 \mathrm{~b}$ shows the microstructure obtained after the heat treatment that has been selected. As expected we have a quite homogeneous microstructure, composed of martensite needles, strained ferrite and bainite.

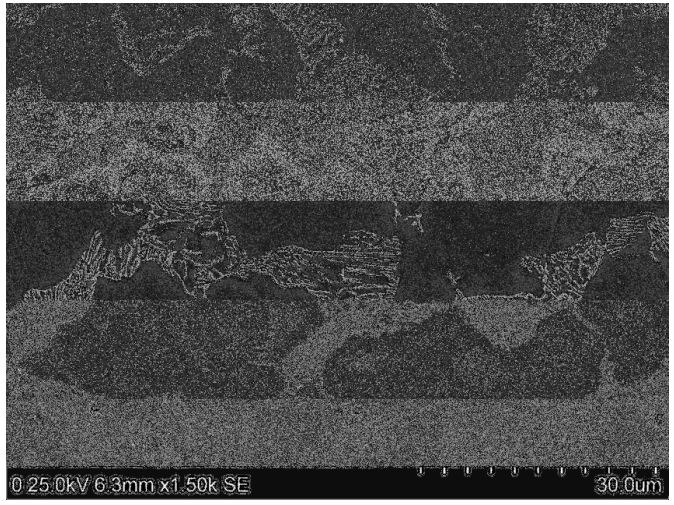

(a)

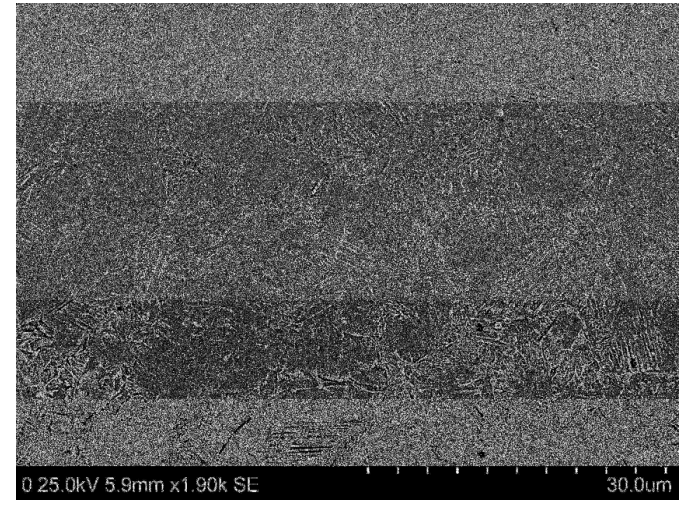

(b)

Fig. 4. Micro graphies of C38 steel: raw material (a), quenched material (b). [15] 


\subsection{Modeling results of DP steels}

Several simulations were carried out using MATLAB 2009 for a dual phase steel. In order to get a reasonable computation time, a fewer number of grains was chosen. On the other hand, the chosen grains should show obvious isotropic transverse symmetry. Computations have been made using a 546 grains RVE of a dual phase steel polycrystal [10].

The experiment results presented herein are performed thanks to a magnetic measurement benchmark where two coils are wounded around the sample. A DP600 from Arcelormittal has been considered for the study in order to underline the effect of the thermo-mechanical history on the magnetic behaviour of these steels. Samples for all experiments consists of $200 \mathrm{~mm}$ long and $12.5 \mathrm{~mm}$ wide bands cut by electro - erosion machining in order to avoid residual stresses that have a strong influence on the magneto - mechanical behavior.

The multiscale modeling allows to predict both the magnetic and magnetostrictive behaviors of the material for different values of the external field and stress. Several samples with different percentage martensite are considered here. Computation for one sample spends almost 48 hours. Results obtained for the anhysteretic magnetization curve are shown in Figure 5a for DP steels with different volume fraction of martensite. Figure $5 \mathrm{~b}$ shows corresponding experimental results for martensite $(100 \%$ Martensite), dual phase (50\%) and fer (0\%) separately.

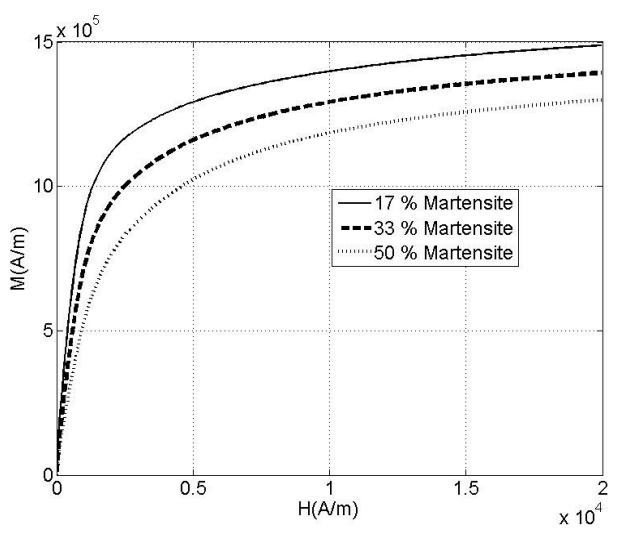

(a) 


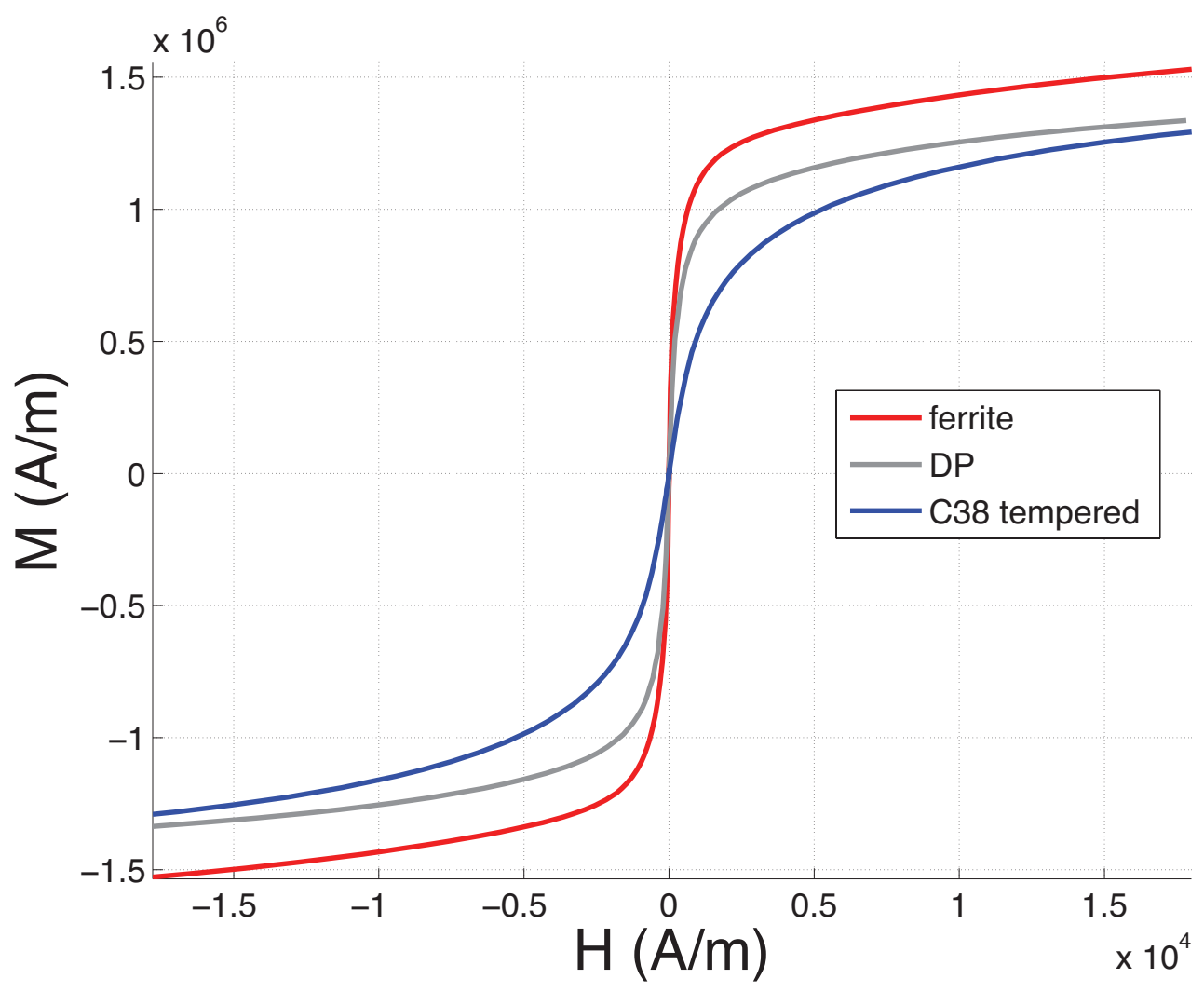

(b)

Fig. 5. Magnetization curve for different volume fractions of martensite: (a) numerical results; (b) experimental results

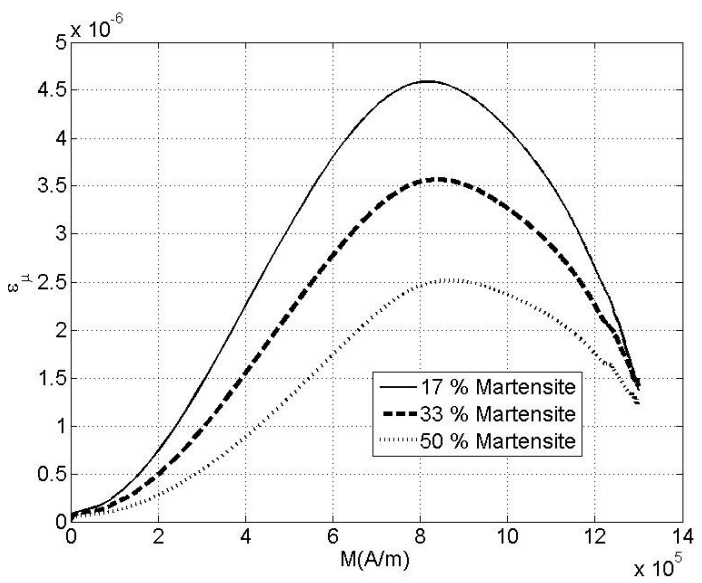

(a) 


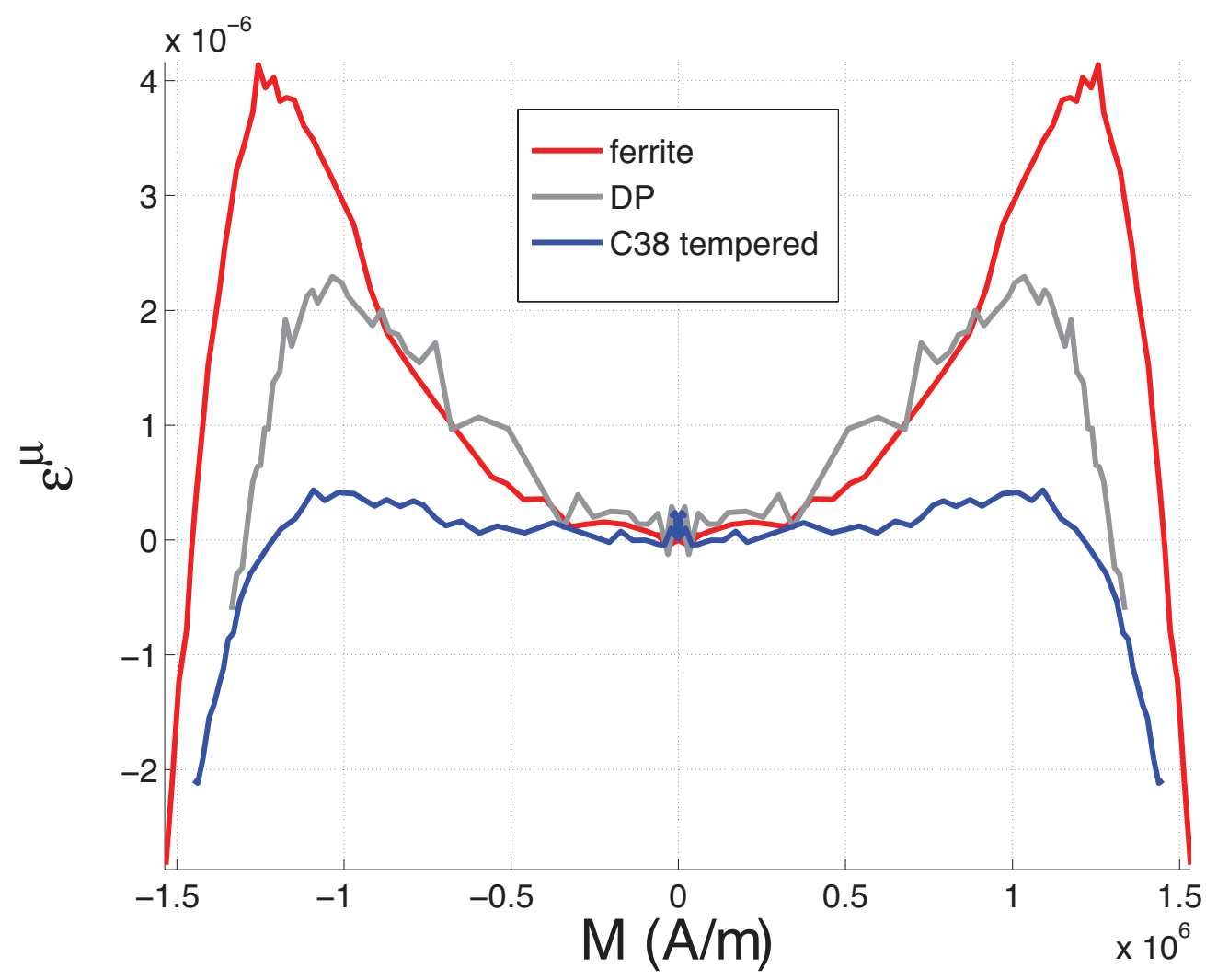

(b)

Fig. 6. Magnetostriction curve for different volume fractions of martensite: (a) numerical results; (b) experimental results

Comparison between experimental and numerical results shows that the multiscale model seems to describe correctly the effect of an applied stress. For the positive magnetic field, a DP steel with lower martensite content has higher magnetic value, and the modeling reproduces it. The result shows that it is more difficult to magnetize the DP steels which contain high volume fractions of martensite Experimental observations (Fig. 6b) show that the magnetostriction strain first increases with the applied field and then decreases until a saturation point is reached. Experimental results illustrate that the DP steel with lower martensite contents has a higher magnetostriction strain, which is correctly reproduced by the numerical result (Fig. 6a).

\section{Conclusion}

In this paper, a model for the reversible magneto-mechanical behavior of magnetic material, accounting for magnetization and microstructure has been presented. It is based on a description of the magneto-mechanical coupling at several scales (domain, single crystal, polycrystal). The modeling strategy allows a fast prediction of the magneto-mechanic behaviour of a dual phase microstructure. This estimation requires the knowledge of the behaviour and the volume fraction of the both phases. Numerical results have been compared with experimental results with very good agreement. 
The main shortcomings of the model are first that magnetic and mechanical fields are considered homogeneous within a single crystal; second that domain walls are not taken into account. Future works will consist in introducing residual stress effects such as plasticity or thermal stress, and dynamic phenomena.

\section{Acknowledgments}

This research was carried out in the frame of the project DPS-MMOD "Dual-Phases Steel Magnetism Modelling" Grant No. MATETPRO 08 - 322447, 2008., funded by the ANR French research agency."

This work is supported by Department of Education of Guizhou Province Foundation (Qian Teaching and Research R\&D [2012] - Grant No. 213), China.

\section{References}

[1] Du Térmolet de Lacheisserie, E.: Magnetostriction-Theory and Applications of Magnetoelasticity. CRC Press, Boca Raton, 1993.

[2] B.D. Cullity, Introduction to magnetic materials, ed. Addison-Wesley, London 1972.

[3] L. Daniel, O. Hubert, EPJ Applied Physics Journal , 45 (2009) 3110-3011.

[4] O. Hubert, L. Daniel, R. Billardon, Przeglad Elektrotechniczny, 81(2005) 19-23.

[5] E Hug, O Huber, M clavel, IEEE Transactions on Magnetics, 33(1997) 763-771.

[6] O. Hubert, L. Daniel, Journal of Magnetism and Magnetic Materials, 304(2006) 489-491.

[7] E. Hug, O. Hubert, J.J. Van Houtte, Materials Science and Engineering, 332(2002) 193-202.

[8] F.S. Mballa, O. Hubert, S. He, S. Depeyre, P. Meilland, IEEE Transactions on Magnetics, (2014) accepted.

[9] L. Daniel, O. Hubert, N. Buiron, R. Billardon, Journal of the Mechanics and Physics of Solids, 56(2008) 1018-1042.

[10] J. D. Eshelby, Proceedings of the Royal Society of London. Series A, Mathematical and Physical Sciences, 241(1957) 376-396.

[11] R.Hill, Journal of the mechanics and physics of solids, 13(1965) 89-101.

[12] M.J. Sablik and D.C. Jiles, IEEE Transactions on Magnetics, 29(1993) 2113-2123.

[13] O. Hubert, L. Daniel, Journal of Magnetism and Magnetic Materials, 323(2011) 1766-1781.

[14] X.J. Wang, Y. Huang, T. Cai, Journal of superconductivity and novel magnetism, 26(2013) 2791-2794.

[15] F.S. Mballa, O. Hubert, S. Lazreg, P. Meilland, 18th World Conference on Nondestructive Testing, (2012). 